\title{
ANÁLISIS Y ESTUDIO DEL DESEMPEÑO DE LOS ENTRELAZADORES DE BITS DE BLOQUE, DORADO Y DORADO RELATIVO EN UN CANAL DE LÍNEA ELÉCTRICA DE BAJA TENSIÓN
}

\section{ANALYSIS OF THE PERFORMANCE OF THE INTERLEAVERS OF BLOCK, GOLDEN AND RELATIVE GOLDEN IN A OF LOW VOLTAGE POWER LINE CHANNEL}

\author{
Washington Fernández R. ${ }^{1} \quad$ Miguel Guzmán U. ${ }^{2} \quad$ Rodrigo Torres S. ${ }^{3}$ \\ Recibido 10 de enero de 2007, aceptado 12 de marzo de 2008 \\ Received: January 10, 2007 Accepted: March 12, 2008
}

\begin{abstract}
RESUMEN
En este trabajo se analizan y estudian tres tipos de entrelazadores de bit: bloque, dorado y dorado relativo, y se determina su desempeño en un canal de línea eléctrica de baja tensión. Se utiliza el modelo de ruido dado por Middleton, para la simulación del canal de línea eléctrica de baja tensión y el algoritmo que se utiliza para generar ruido gaussiano es el método de Box-Muller. Se compara el desempeño de los entrelazadores de bits de bloque, dorado y dorado relativo, en relación al largo de la palabra versus la razón de señal a ruido y bits erróneos por palabras versus la razón de señal a ruido. La codificación de línea que se utiliza es no retorno a cero. El entrelazador de bit dorado es el que tiene el mejor desempeño, por lo tanto, se puede emplear en la transmisión por línea eléctrica de baja tensión para disminuir los errores de bits consecutivos producidos por el ruido impulsivo.
\end{abstract}

Palabras clave: Entrelazador de bit dorado, dorado relativo, ruido en línea eléctrica de baja tensión.

\begin{abstract}
This paper analyzes three types of interleavers: block, golden and golden relative to determine their performance in of low voltage power line channel. The model of noise given by Middleton is used for the simulation of the low voltage power line channel and the algorithm used to generate Gaussian noise is based on the Box-Muller method. The performance of interleavers block, golden and golden relative is compared in relationship to the length of the words versus the signal to noise ratio. The error bit by word versus signal to noise ratio is compared also. The codification of line used is no return to zero. It is concluded that the interleavers of golden has the best performance, thus it can be used in data transmission in low-voltage power line, reducing errors consecutive bits produced by impulse noise.
\end{abstract}

Keywords: Interleavers, golden, golden relative, noise in low voltage power line.

\section{INTRODUCCIÓN}

Los códigos de corrección de errores funcionan adecuadamente con tal de que no haya demasiados errores de bits en una sola palabra de código. Sin embargo, los errores muchas veces son del tipo de ráfaga en el sentido de que existe una concentración de muchos errores en una palabra de código, es decir, demasiados para manejar la corrección de estos.

Específicamente esta situación ocurre en canales de comunicaciones móviles y en los sistemas que utilizan como canal de transmisión la línea eléctrica de baja tensión.

El entrelazador de bit toma la secuencia de símbolos de un alfabeto fijo y produce una secuencia de salida del mismo alfabeto que es idéntico a la sucesión de la entrada salvo en el orden de la secuencia. Cada entrelazador de bit tiene su correspondiente desentrelazador de bit, el cual vuelve la secuencia de símbolos a su orden original, el primero ubicado en el transmisor y el segundo ubicado en el receptor. Existe una gran variedad de tipos entrelazadores de bit [1-2]; se tienen: entrelazador de bit aleatorio [3-4], entrelazador

\footnotetext{
1 Departamento de Ingeniería Eléctrica y Electrónica. Universidad del Bío-Bío. Avenida Collao 1202. Concepción, Chile. E-mail: wfernand@ ubiobio.cl

2 Departamento de Ingeniería Eléctrica y Electrónica. Universidad del Bío-Bío. Avenida Collao 1202. Concepción, Chile. E-mail: mguzman@ubiobio.cl

3 Departamento de Ingeniería Eléctrica y Electrónica. Universidad del Bío-Bío. Avenida Collao 1202. Concepción, Chile. E-mail: rtorres@ubiobio.cl
} 
de bit algebraico, [5-6], entrelazador de bit exploración helicoidal [7], entrelazador de bit multiplexado general y entrelazador de bit difundido [8-10]. El entrelazador de bits que ha sido más investigado es el entrelazador aleatorio o también denominado entrelazador "expandido" [11-13]. El entrelazador de bit aleatorio realiza una permutación aleatoria o seudoaleatoria de los elementos sin ninguna restricción. Un número de variaciones de los entrelazadores aleatorios se analizan en [14-17].

Un problema con la transmisión en el canal de línea eléctrica de baja tensión es que se generan errores de ráfaga de longitud variable. Esto hace muy difícil para el diseño de entrelazadores de bits óptimo cuando se usan los métodos clásicos. El objetivo es entrelazadores de bits que sean buenos para cualquier longitud de errores de ráfagas.

El entrelazador de bit dorado [18-19] da una solución a este problema. Por esta razón, que se estudia su desempeño en un canal de línea de baja tensión.

La comunicación PLC (PowerLine Communications: Línea eléctrica de comunicación) utiliza las líneas eléctricas de baja tensión (220-380 V) como un canal de transmisión de datos, pero la red de energía eléctrica no se diseña para este fin; el canal presenta muchas desventajas que impiden una buena transmisión. La mayor ventaja con este tipo de transmisión es que la cobertura de la red eléctrica en Chile es de 96,1\% de la población, muy superior al 51,5\% de cobertura de la red de telefonía fija. Las desventajas asociadas con la transmisión sobre líneas de energía son los altos niveles de ruido, alta atenuación, distorsión de la señal y la imposibilidad de que señales de frecuencias altas pasen por los transformadores de distribución, ya que éstos presentan una alta impedancia y característica de filtro pasa bajo. La red de distribución de energía eléctrica es, por lo anterior, un medio extremadamente hostil como canal de comunicación [20].

La línea eléctrica de baja tensión presenta cinco diferentes clases de ruido [20]:

Ruido no blanco de fondo.

Ruido de banda estrecha.

Ruido impulsivo periódico-asíncrono con la frecuencia de la red.

Ruido impulsivo periódico-síncrono con la frecuencia de la red.

Ruido impulsivo asíncrono.

Middleton en 1977 [21] plantea un modelo de ruido impulsivo, el cual está formado por la suma de ruido blanco y ruido impulsivo. Según el ancho de banda del ruido, se clasifican en tres clases generales: clase $\mathrm{A}$, clase B y clase $\mathrm{C}$.

Ruido clase A: Es la interferencia cuyos espectros son iguales o más estrechos que el ancho de banda del receptor.

Ruido clase B: Son los ruidos cuyos espectros son más amplios que el ancho de banda del receptor.

Ruido clase C: Son las interferencias que incluyen las características de clase A y B.

De acuerdo a [21], el ruido presente en las líneas eléctricas es del tipo clase A.

\section{ANÁLISIS TEÓRICO}

\section{Descripción del sistema}

La sección dorada es un problema matemático muy interesante. La figura 1 ilustra el principio de la sección dorada en relación al problema de entrelazador de bit. Se da un segmento de línea de largo 1, el problema es dividirlo en un segmento de largo g y un segmento más corto de largo 1-g tal que la razón entre el segmento más largo es al segmento completo es la misma, como la proporción entre el segmento más corto es al segmento más largo.

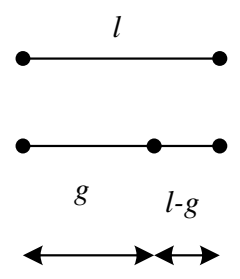

Figura 1. Ilustración del principio sección dorada, para aplicarlo al entrelazador de bit dorado.

De acuerdo a la figura 1, la ecuación es:

$$
\begin{aligned}
& \frac{g}{l}=\frac{l-g}{g} \\
& g^{2}-l+l g
\end{aligned}
$$

Resolviendo esta ecuación de segundo orden se tiene:

$$
g=-\frac{1}{2} \pm \frac{\sqrt{l^{2}+4 l}}{2}
$$


Para el caso particular de $1=1$, se tiene:

$$
g=\frac{\sqrt{5}-1}{2}=0.618
$$

Para el entrelazador de bit dorado relativo como para el entrelazador de bit dorado, en el primer paso se determina el valor de $g$.

Posteriormente se calcula el incremento real relativo, el cual se denomina $c$ y se define como:

$$
c=\frac{N\left(g^{m}+j\right)}{r}
$$

Donde $g$ es el valor dorado y $m$ es cualquier número entero positivo mayor que cero; los valores adecuados para $m$ son típicamente 1 o 2, $r$ es el esparcimiento de la tabla (la distancia que deja el entrelazador de bit a los bits consecutivos) y $j$ es cualquier módulo entero de $r$. Después se obtiene el incremento primo relativo $p$, tomando el valor entero más cercano a $c$. Posteriormente se calculan los índices del entrelazador de bit como sigue:

$i(n)=s+n p \quad$ módulo $N, \quad n=0,1, \ldots \ldots, N-1$

Se define la operación módulo como: es el resto en $\{0$, ..., $N-1\}$, de la división de $a$ por $n$. Por ejemplo: 23 módulo $12=11$.

Donde $s$ es un índice de comienzo, $p$ es el incremento del índice y $N$ es el largo del entrelazador de bit. Los $N$ y $p$ son primos relativos, los cuales aseguran que cada elemento se muestre en la salida una y sólo una vez. El índice $s$ es el de comienzo, se le asigna generalmente el valor 0 , pero también cualquier valor entero es válido. Para el entrelazador de bit dorado el primer paso calcula el valor dorado $g$. El segundo paso determina el valor real de incremento $c$, que es igual al entrelazador de bit dorado relativo. El tercer paso genera el vector dorado de valor real $\boldsymbol{v}$. Los elementos de $v$ se determinan como sigue:

$$
\boldsymbol{v}(n)=s+n c \quad \text { módulo } N, \quad n=0,1, \ldots \ldots, N-1
$$

Donde $s$ es cualquier valor inicial real. El siguiente paso ordena el vector dorado $\boldsymbol{v}$ y encuentra el vector $z$, de los índices. Se encuentra y ordena $z$ vectorial, tal que:

$$
\begin{gathered}
\mathbf{a}(n)=\mathbf{v}[\mathbf{z}(n)] n=0,1, \ldots \ldots, N-1 \\
\boldsymbol{a}=\operatorname{sort}(\mathbf{v})
\end{gathered}
$$

Donde sort es una función que ordena una matriz de menor a mayor. Los índices del entrelazador de bit dorado son dados por:

$$
i[z(n)]=n \quad n=0,1, \ldots \ldots \ldots, N-1
$$

El valor inicial de $s$ es 0 , pero cualquier valor real también sirve. Los valores adecuados para $m$ son típicamente $1 \mathrm{o}$ 2. Para la dispersión máxima de elementos adyacentes, $j$ toma el valor 0 y $r$ asume valor 1 .

Ejemplo:

La secuencia de bit es $\left[\begin{array}{lll}0 & 12345678910111213\end{array}\right.$ 14]. Se quiere que los elementos colindantes queden lo más separado posible uno del otro, se elige $r=1, j=0$, $m=1$ y $s=0$. Se calcula:

$$
\begin{aligned}
& g=\frac{\sqrt{5}-1}{2}=0.618 \\
& c=\frac{N\left(g^{m}+j\right)}{r}=\frac{15(0.618+0)}{1}=9 . .27 \\
& \boldsymbol{v}(n)=(s+n c) \text { módulo } N \quad n=0,1, \ldots \ldots, N-1 \\
& \boldsymbol{v}(n)=(9.27 n) \text { módulo } 15 \\
& \boldsymbol{v}=(0,9.27,3.54,12.81,7.08,1.35,10.62,4,89 \\
& \quad 14.16,8.43,2.7,11.97,6.24,0.51,9.78)
\end{aligned}
$$

Se determina el vector:

$$
\mathbf{a}(n)=\mathbf{v}[\mathbf{z}(n)]
$$

Se ordena el vector $\boldsymbol{v}$, de menor a mayor:

$$
\begin{aligned}
& \boldsymbol{a}=(0,0.51,1.35,2.7,3.54,4.89,6.24,7.08,8.43, \\
& \quad 9.27,9.78,10.62,11.97,12.8,14.16) \\
& \boldsymbol{a}=\boldsymbol{v}(0,13,5,10,2,7,12,4,9,1,14,6,11,3,8) \\
& i[z(n)]=n \\
& i=0,13,5,10,2,7,12,4,9,1,14,6,11,3,8)
\end{aligned}
$$

El ruido del canal de línea eléctrica de baja tensión se expresa por una componente en fase y una componente en cuadratura como [21]:

$$
n(t)=X(t) \cos \omega_{c} t+Y(t) \operatorname{sen} \omega_{c} t
$$

donde:

$n(t)$ : Ruido del canal de línea de tensión de baja tensión.

$X(t)$ : Componente en fase del ruido.

$Y(t)$ : Componente en cuadratura de fase del ruido.

$f c \quad$ : Frecuencia central del ruido. 
El modelo de Middleton [21] se representa por el producto de una distribución Poisson, $P(m ; A)$ y una distribución de Gauss, $G\left(z ; \sigma_{m}^{2}\right)$. Esto es:

$$
p_{A}(z)=\sum_{m=0}^{\infty} P(m ; A) G\left(z ; \sigma_{m}^{2}\right)
$$

donde:

$P_{A}(z) \quad$ : Función de densidad de probabilidad.

$z \quad$ : Variable aleatoria de ruido.

$\sigma_{m}^{2} \quad$ : Varianza de Middleton.

$m \quad$ : Número de subcanales de Middleton.

A : Índice impulsivo.

$$
\begin{gathered}
P(m ; A)=\frac{e^{-A} A^{m}}{m !} \\
G\left(z ; \sigma_{m}^{2}\right)=\frac{1}{\sqrt{2 \pi \sigma_{m}^{2}}} e^{-\frac{\left|z^{2}\right|}{2 \sigma_{m}^{2}}}
\end{gathered}
$$

El parámetro $A$ se define por el producto entre la duración promedio de los impulsos y la razón de generación media del ruido impulsivo (cantidad de eventos impulsivos por unidad de tiempo).

La varianza de Middleton se obtiene [21] como:

$$
\sigma_{m}^{2}=\sigma^{2} \frac{\frac{m}{A+\Gamma}}{1+\Gamma}
$$

donde:

$\Gamma$ : Relación entre las potencias gaussiana e impulsiva, y es dada por [21]:

$$
\Gamma=\frac{\sigma_{G}^{2}}{\sigma_{i}^{2}}
$$

El modelo de Middleton clase A consiste de la sumatoria de infinitos ruidos gaussianos, cada uno con distinta potencia de ruido. Aquellos que presentan mayor potencia se denominan impulsivos y los que tienen una potencia de ruido mucho menor (al menos 10 veces, según [21]), se definen como gaussianos. La distribución de Poisson representa la probabilidad de un ruido más impulsivo a uno menos impulsivo y viceversa.

De acuerdo [21], la potencia de ruido gaussiano y su varianza se relacionan por:

$$
N_{G}=2 \sigma_{G}^{2}
$$

Como el modelo de Middleton se compone de ruido gaussiano y ruido impulsivo, ambos con una distribución de Gauss, se cumple que:

$$
N_{o}=N_{G}+N_{i}=2 \sigma_{G}^{2}+2 \sigma_{i}^{2}=2 \sigma
$$

donde:

$N_{O}$ : Densidad de potencia de ruido de Middleton. $N_{G}$ : Densidad de potencia de ruido gaussiano.

$N_{i}$ : Densidad de potencia de ruido impulsivo.

La varianza del ruido en la línea eléctrica de baja tensión se representa por $\sigma$ y es la suma de las varianzas de la componente gaussiana y la componente impulsiva. Es decir,

$$
\sigma=\sigma_{G}^{2}+\sigma_{i}^{2}
$$

donde:

$\sigma^{2} \quad$ : Varianza del ruido de línea eléctrica de baja tensión.

$\sigma_{G}^{2} \quad$ : Varianza de la componente gaussiana del ruido.

$\sigma_{i}^{2} \quad$ : Varianza de la componente impulsiva del ruido.

Las componentes normalizadas $\mathrm{x}(\mathrm{t})$ e $\mathrm{y}(\mathrm{t})$ de las componentes $\mathrm{X}(\mathrm{t})$ e $\mathrm{Y}(\mathrm{t})$ de la ecuación (11) se obtienen al dividir estas últimas por la varianza del ruido $\sigma$; de acuerdo a [22] se tiene:

$$
\begin{aligned}
& x(t)=\frac{X(t)}{\sqrt{\sigma_{G}^{2}+\sigma_{i}^{2}}} \\
& y(t)=\frac{Y(t)}{\sqrt{\sigma_{G}^{2}+\sigma_{i}^{2}}}
\end{aligned}
$$

El algoritmo que se utiliza para la simulación del ruido de línea eléctrica de baja tensión incluye dos subcanales equiprobables de Middleton. La variable $\sigma_{G}^{2}$ representa la varianza del ruido gaussiano de fondo y $\sigma_{i}^{2}$ (10 veces mayor a $\sigma_{G}^{2}$ ), la varianza del subcanal impulsivo. Como ambos subcanales presentan una distribución de Gauss, se emplea el algoritmo de Box-Muller [23-25], con:

$x_{1}$ : Variable con distribución uniforme $[0,1]$.

$x_{2}$ : Variable con distribución uniforme $[0,1]$. 


$$
a=\sqrt{-\ln x_{1}} \cos 2 \pi x_{2}
$$

Para este caso particular el algoritmo Box-Muller es:

$x(t)=$ Variable aleatoria con distribución uniforme $[0,1]$. $y(t)=$ Variable aleatoria con distribución uniforme $[0,1]$.

$$
n_{G}=\sqrt{\sigma_{G}^{2}\{-\ln [x(t)]\}} \cos [2 \pi y(t)]
$$

$n_{G}$ : Componente gaussiana del ruido.

$$
n_{i}=\sqrt{\sigma_{i}^{2}\{-\ln [x(t)]\}} \cos [2 \pi y(t)]
$$

$n_{i}$ : Componente impulsiva.

Se utilizan las ecuaciones (23) y (24), porque permiten la simulación de la distribución de Gauss con una mejor exactitud [24-25].

En este trabajo se determina el desempeño de los entrelazadores de bits del tipo dorado y dorado relativo en un canal de línea eléctrica de baja tensión, considerando que este canal tiene un ruido predominantemente impulsivo, los cuales son ráfagas de corta duración, es decir, de gran amplitud y de corta duración aleatorios, provocando con esto que varios bits consecutivos de una palabra lleguen erróneos al receptor. La función del entrelazador de bit es dispersar los bits consecutivos en diferentes posiciones de la palabra en el bloque que se transmite. La codificación de línea que se utiliza es no retorno a cero. Como referencia de comparación del desempeño de los entrelazadores dorado y dorado relativo es el entrelazador de bloque. La figura 2 muestra el diagrama de bloque del sistema de transmisión y recepción propuesto.

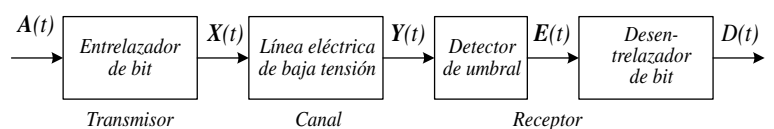

Figura 2. Sistema de transmisión y recepción propuesto.

De acuerdo a la figura 2, el transmisor se representa por el entrelazador de bit, el receptor por el detector de umbral y el desentrelazador de bit.

La salida $\boldsymbol{A}(t)$ representa los bits de datos que se transmiten, y tienen representación de no retorno a cero; estos pasan por el entrelazador de bit, que cambia de orden los bits de los datos que se transmiten $\boldsymbol{X}(t)$ (según sea el entrelazador que se use: bloque, dorado o dorado relativo). Esta señal es la que se transmite a través del canal de línea eléctrica de baja tensión, por lo tanto, la señal transmitida se contamina con el ruido del canal y se recibe como $\boldsymbol{Y}(t)$ :

$$
\boldsymbol{Y}(t)=\boldsymbol{X}(t)+N(t)
$$

donde:

$\boldsymbol{Y}(t)$ : Señal que se recibe.

$\boldsymbol{X}(t)$ : Señal que se transmite.

$N(t)$ : Ruido del canal de línea eléctrica de baja tensión.

En el receptor se usa un detector básico (detector de umbral), que recupera los datos que se transmiten, $\boldsymbol{E}(t)$. El criterio de decisión es: valores mayores e iguales que 0.5 corresponden al " 1 " y valores menores que 0.5 , corresponden al " 0 ". El desentrelazador de bit retorna a la ubicación original los bits transmitidos $\boldsymbol{D}(t)$. Después comparando $\boldsymbol{A}(t) \operatorname{con} \boldsymbol{D}(t)$ se determina el número de palabras erróneas y la cantidad de bits erróneos por palabra.

\section{RESULTADOS}

La figura 3 muestra el resultado de la simulación del ruido del canal de línea eléctrica de baja tensión. La parte superior muestra el ruido de componente gaussiana, y abajo la componente de carácter impulsiva.

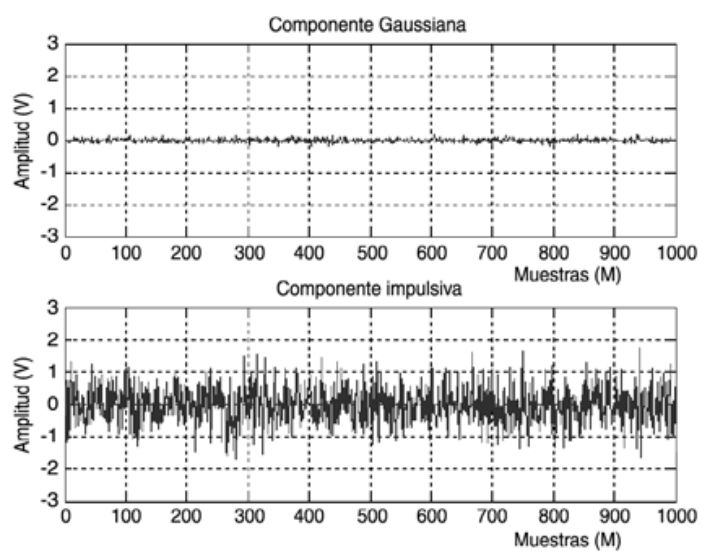

Figura 3. Simulación del ruido del canal de línea eléctrica de baja tensión: arriba componente gaussiana, abajo componente impulsiva.

La figura 4 muestra razón de palabras erróneas en función del SNR para el entrelazador de bit de bloque con largo de palabra de 20 bits, tamaño de bloque de 1.000, 2.000 y 5.000 bits. 


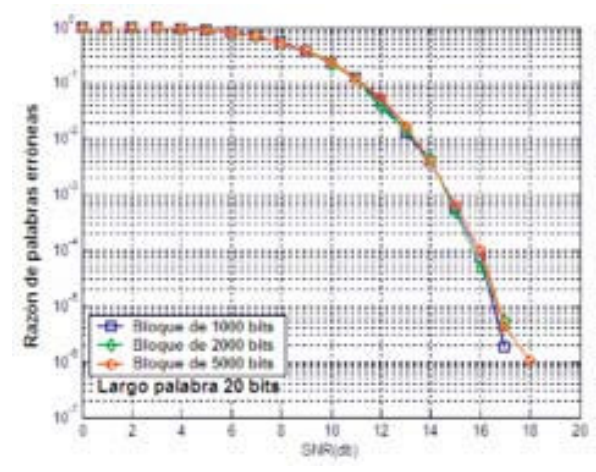

Figura 4. Razón de palabras erróneas en función de SNR para entrelazador de bit de bloque con tamaño de palabra de 20 bits y tamaño de bloque 1.000 , 2.000 y 5.000 bits.

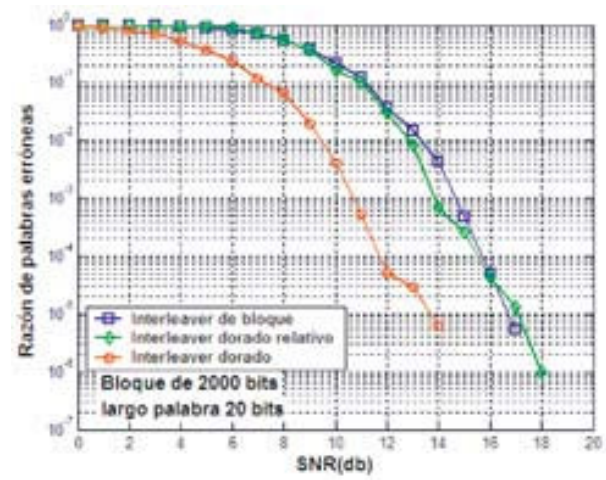

Figura 6. Comparación del desempeño de los entrelazadores de bloque, dorado relativo y dorado, con tamaño de bloque de 2.000 bits y tamaño de palabra de 20 bits.

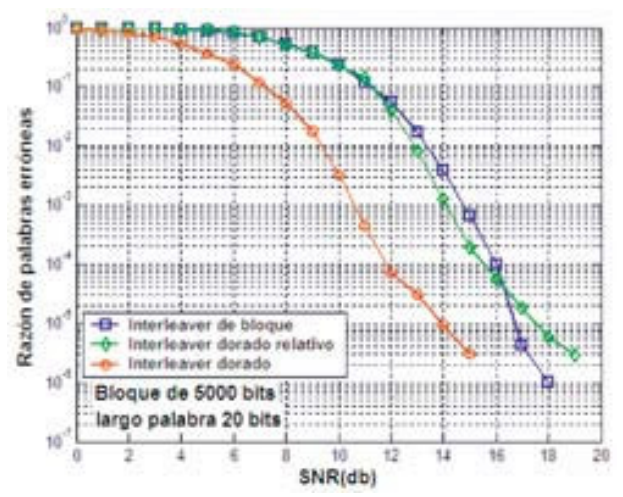

Figura 8. Comparación del desempeño de los entrelazadores de bloque, dorado relativo, con tamaño de bloque de 5.000 bits y tamaño de palabra de 20 bits.

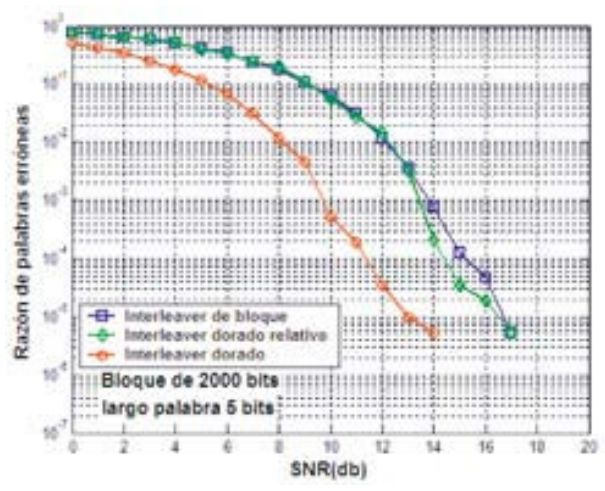

Figura 5. Comparación del desempeño de los entrelazadores de bloque, dorado relativo y dorado, con tamaño de bloque de 2.000 bits y tamaño de palabra de 5 bits.

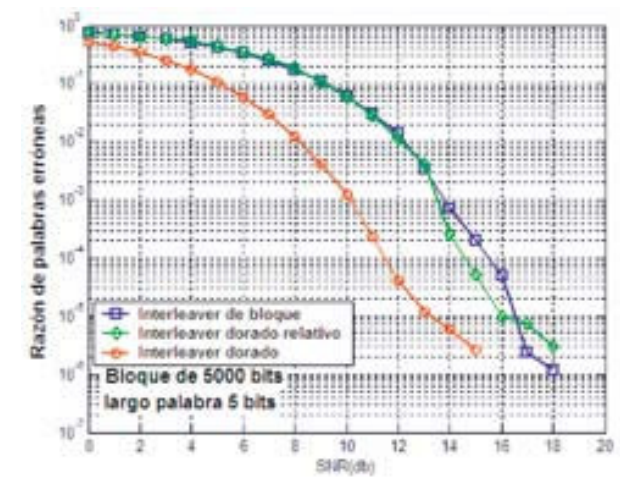

Figura 7. Comparación del desempeño de los entrelazadores de bloque, dorado relativo y dorado, con tamaño de bloque de 5.000 bits y tamaño de palabra de 5 bits.

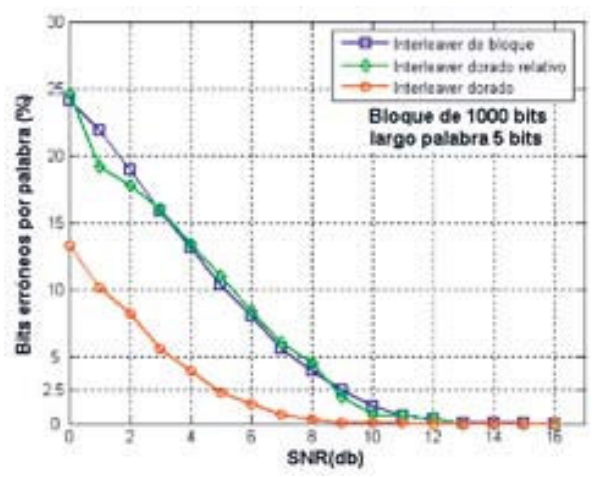

Figura 9. Porcentaje de bits erróneos por palabra en función de SNR, para los entrelazadores de bits de bloque, dorado relativo y dorado, con tamaño de bloque de 1.000 bits y tamaño de palabra de 5 bits. 
Las figuras 5, 6, 7 y 8 comparan el desempeño de los entrelazadores de bits de bloque, dorado y dorado relativo con tamaño de bloque de $2.000,5.000$ bits y tamaño de palabras de 5 y 20 bits.

Las figuras 9, 10, 11 y 12 muestran el porcentaje de bits erróneos por palabra en función de SNR, para los entrelazadores de bits de bloque, dorado relativo y dorado, con tamaño de bloque de $1.000,5.000$ bits y tamaño de palabra 5 y 20 bits.

\section{DISCUSIÓN DE LOS RESULTADOS}

De la figura 1, la simulación del ruido para una línea eléctrica de baja tensión tiene la misma respuesta que el reportado por la literatura $[20,22]$.

La figura 4 muestra que el desempeño del entrelazador de bloque con largo de palabra de 20 bits y tamaño de bloque $1.000,2.000$ y 5.000 bits, no presenta una gran diferencia del desempeño con relación al tamaño del bloque.

De las figuras 5, 6, 7 y 8, donde se compara el desempeño de los entrelazadores de bits de bloque, dorado y dorado relativo con tamaño de bloque de $2.000,5.000$ bits y tamaño de palabras de 5 y 20 bits, se observa que el entrelazador de bit dorado es el de mejor desempeño, considerando como parámetro la razón de palabras erróneas versus SNR. Se tiene, por ejemplo, una ganancia mayor a $3 \mathrm{~dB}$ del entrelazador de bit dorado con respecto al entrelazador de bit dorado relativo, para obtener un BER de $1 \times 10^{-5}$.

De acuerdo a los resultados de las figuras 9, 10, 11 y 12 , se tiene que para el parámetro porcentaje de bits erróneos por palabra versus SNR, considerando el largo de la palabra y tamaño del bloque, el de mejor desempeño es el entrelazador de bit dorado.

De acuerdo a los resultados obtenidos, el entrelazador de bit que presenta un mejor desempeño, en un canal de línea eléctrica de baja tensión, es el dorado, considerando razón de palabras erróneas versus SNR y porcentaje de bit erróneos por palabra versus SNR.

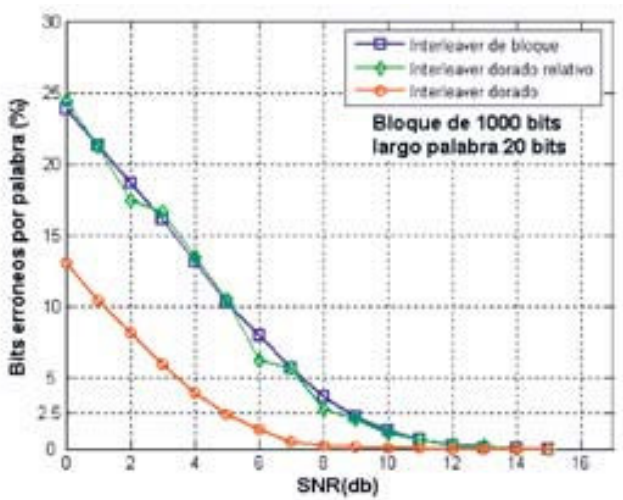

Figura 10. Porcentaje de bits erróneos por palabra en función de SNR para los entrelazadores de bits de bloque, dorado relativo y dorado, con tamaño de bloque de 1.000 bits y tamaño de palabra de 20 bits.

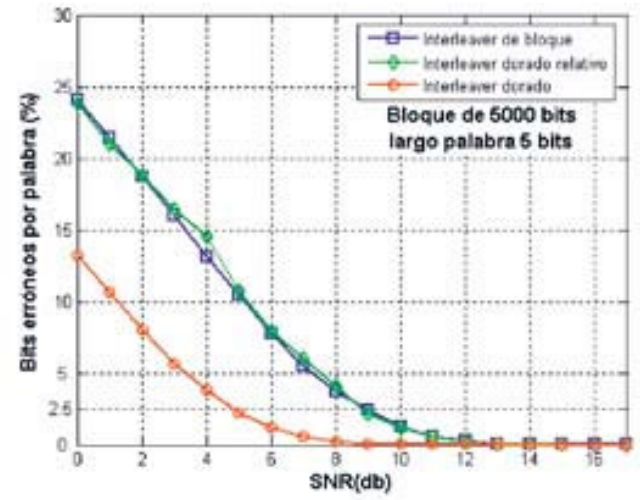

Figura 11. Porcentaje de bits erróneos por palabra en función de SNR para los entrelazadores de bits de bloque, dorado relativo y dorado, con tamaño de bloque de 5.000 bits y tamaño de palabra de 5 bits.

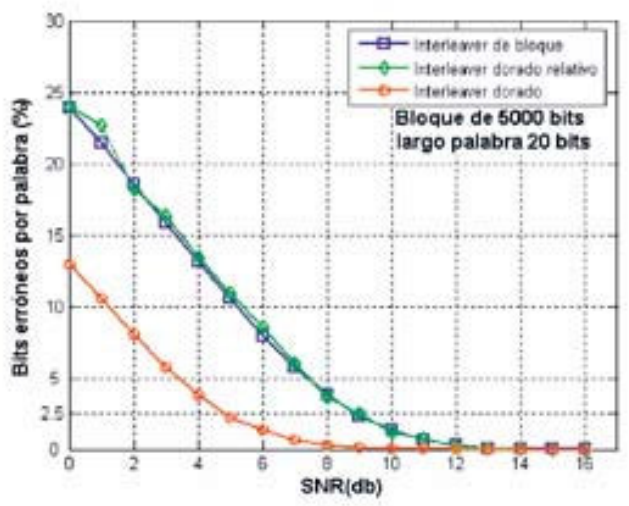

Figura 12. Porcentaje de bits erróneos por palabra en función de SNR para entrelazadores de bits de bloque, dorado relativo y dorado, con tamaño de bloque de 5.000 bits y tamaño de palabra de 20 bits. 


\section{CONCLUSIONES}

De la discusión de los resultados obtenidos de la simulación, el entrelazador de bit dorado es el que tiene el mejor desempeño, por lo tanto, se puede emplear en la transmisión por línea eléctrica de baja tensión, para disminuir los errores de bits consecutivos producidos por el ruido impulsivo. Como futuro trabajo de investigación, está el estudiar el desempeño de la combinación de códigos de chequeo de baja densidad irregular con entrelazador de bit dorado en un canal de línea eléctrica de baja tensión, la razón es porque los códigos de chequeo de paridad de baja densidad tienen un muy buen desempeño en la detección y corrección de errores.

\section{AGRADECIMIENTOS}

Los autores de este trabajo agradecen a la Universidad del Bío Bío, Concepción-Chile, por apoyar y financiar el proyecto de investigación código 052210 3/R.

Los autores también están muy agradecidos por los comentarios y observaciones de los revisores, lo cual ha permitido enriquecer este artículo.

\section{REFERENCIAS}

[1] D. Divsalar and F. Pollara. "Multiple Turbo Codes for Deep-Space Communications". JPL, TDA Progress Report 42-121. May 15, 1995.

[2] S. Benedetto and G. Montorsi. "Unveiling Turbo Codes: Some Results on Parallel Concatenated Coding Schemes". IEEE Transactions on Information of Theory, Vol. $42 \mathrm{~N}^{\circ} 2$, pp. 409-428. March 1996.

[3] G. Battail. "On random like codes". Proceeding $4^{\text {th }}$ Canadian Workshop on information theory. Lac Delage, Quebec, Canada. Mayo 1995.

[4] J. Yuan and B. Vucetic. "Combined turbo code and interleaver design". IEEE Transactions on Communications. Vol. 47 No 4, pp. 484-487. April 1999.

[5] O. Takeshita. "Permutation polynomial interleavers: Algebraic construction perspective, URLs: www. arviv.org/abs/cs/2601948/2006

[6] O. Takeshita and D. Costello. "New classes of algebraic interleavers for turbo code". Proceeding 1998. IEEE Symposium on information theory. Boston. USA. August 1998.
[7] O. Takeshita and D. Costello. "On deterministic linear interleavers for turbo code". Proceeding of $35^{\text {th }}$ annual Allerton conference on communication control and computing. September 1997.

[8] G. Forney. "Interleavers". USA patent 3.652.998. URLs: www.freepatentonline.com/3.652.998. pdf

[9] M. Fimmoff. "Convolutional interleaver and deinterleavers". USA patent: 5.572.532. URLs: www.freepatentonline.com/5.572.532.pdf

[10] M. Prasad. "Interleavers and de-interleavers", USA patent: 6.748.561B2. URLs: www.freepatentonline. com/6.748.561B2.pdf

[11] S. Benedetto and G. Montorsi. "Tutorial 11: Turbo Codes: Comprehension, Performance Analysis, Design, Iterative Decoding”. IEEE International Conference on Communications (ICC'97). Montreal, Quebec, Canada. June 8-12, 1997.

[12] A. Barbulescu and S. Pietrobon. "Interleaver Design for Turbo Codes". Electronics Letters, Vol. 30 N $^{\circ}$ 25, pp. 2107-08. December 8, 1994.

[13] M. Ho, S. Pietrobon and T. Giles. "Interleavers for Punctured Turbo Codes". IEEE Asia-Pacific Conference on Communications. (APCC'98) and Singapore Int. Conference on Communications Systems. Vol. 2, pp. 520-524. Singapore. November, 1998.

[14] S. Pietrobon. "Interleaver Address Generator". Version 1.01. October 4, 1998. Available from Small World Communications. URLs: www.sworld.com.au

[15] P. Sauvé. "Multibit Decoding of Turbo Codes". Master's Thesis. University of Toronto. Canada. October 1998.

[16] J.L. Ramsey. "Realization of optimum Interleavers". IEEE Transactions on Information Theory. Vol. IT-16 No 3, pp. 338-345. May 1970.

[17] B. Sklar. "Digital Communications: Fundamentals and Application". Second Edition. Prentice Hall. 2001.

[18] S. Crozier and J. Lodge. "Performance of Turbo Codes with Relative Prime and Golden Interleaving Strategies". URLs: www.crc.ca/fec 
[19] R. Garello and G. Montorsi. "Interleaver properties and their applications to the trellis complexity analysis turbo codes". IEEE transactions on Communications. Vol. $49 \mathrm{~N}^{\circ}$ 5. May 2002, pp. 793807.

[20] M. Zimmermann and K. Dostert. "Analysis and Modeling of Impulsive noise in Broad-Band Powerline Communications". IEEE Transactions on Electromagnetic Compatibility. Vol. $44 \mathrm{~N}^{\mathrm{o}} 1$, pp. 249-258. February 2002.

[21] D. Middleton. "Canonical Non Gaussian Models: Their Implications for Measurements and Prediction Receiver Performance". IEEE Transactions of Communications. Vol. COM-21 No 3, pp. 209-220. August 1979.

[22] M. Katayama and T. Yamazato. "A mathematical model of noise in narrowband power line communications system". IEEE Journal on Selected areas in Communications. Vol. $24 \mathrm{~N}^{\mathrm{o}} 7$, pp. 1267-1276. July 2006.

[23] W. Fernández. "Combinación de códigos LDPC irregulares con Canal MIMO para la Cuarta Generación 4G". Tesis para optar al grado de magíster. Departamento de ingeniería eléctrica. Universidad de Santiago de Chile. Santiago, Chile. 2003.

[24] G. Box and M. Muller. "A note on the generation of random normal deviates". Annals mathematic statistics. Vol. 29 No 2, pp. 610-611. 1958.

[25] D. Lee and J. Villasenor. "A hardware Gaussian noise generator using the Box-Muller method and its error analysis". IEEE Transactions on Computer. Vol. 55 Issue 6, pp. 659-671. June 2006. 